\title{
Online identification of Thevenin equivalent circuit model parameters and estimation State Of Charge of Lithium-Ion batteries
}

\author{
Edoardo Locorotondo, Luca Pugi, Lorenzo Berzi, Marco Pierini \\ Department of Industrial Engineering \\ University of Florence \\ Florence, Italy \\ edoardo.locorotondo@unifi.it, luca.pugi@unifi.it, \\ lorenzo.berzi@unifi.it, marco.pierini@unifi.it
}

\author{
Giovanni Lutzemberger \\ Department of Energy and Systems Engineering \\ University of Pisa \\ Pisa, Italy \\ lutzemberger@dsea.unipi.it
}

\begin{abstract}
On Electrical and Hybrid Vehicles (EVs, HEVs), energy is stored in accumulators, mainly electro-chemical batteries. A reliable and cost effective management of energy storage system is a key point for the development of such devices, their durability and for vehicle performance optimization. This requires the accurate estimation of the battery state over time and in a wide range of operating conditions. The battery state is usually expressed as State Of Charge (SOC) and State Of Health (SOH). Their estimations requires an accurate model to represent the static and dynamic behaviors of the battery. This paper presents a model adaptive Unscented Kalman Filter (UKF) method to estimate online SOC of Li-ion batteries. The proposed approach uses a Recursive Least Squares method to update the UKF model parameters during a discharge period. The effectiveness of the method has been verified based on real data acquired from five $\mathrm{LiFePO} 4$ battery packs installed on a working EV.
\end{abstract}

Keywords-battery; lithium-ion; SOC; Unscented Kalman Filter; parameter identification; adaptive model; electric vehicles;

\section{INTRODUCTION}

The development of electrical mobility systems is a fundamental topic for european research project, for example "Smart, Green and Integrated Transport" in Horizon 2020. Monitoring and management of energy accumulators (rechargeable batteries) for EV is one of the main themes of research and industrial development. Smart energy management defines the relationship between cost and quality of EV; it is fundamental to define weight, reduction of space, and in conclusion an appropriate safety level. Today, lithium batteries have almost replaced the lead acid batteries. Indeed a lithium cell should accumulate an higher energy and it should deliver an higher power than lead acid cells. Furthermore lithium cells have a longer cycle life and have a better environmental impact than lead acid cells. Battery Management System (BMS) is the main control system: it is usually used to enhance lithium battery performance and to work in recommended SOC window, consequently to avoid over-voltages and under-voltages and to work in its safe operating temperature range. To improve battery state estimation accuracy, BMS should be able to

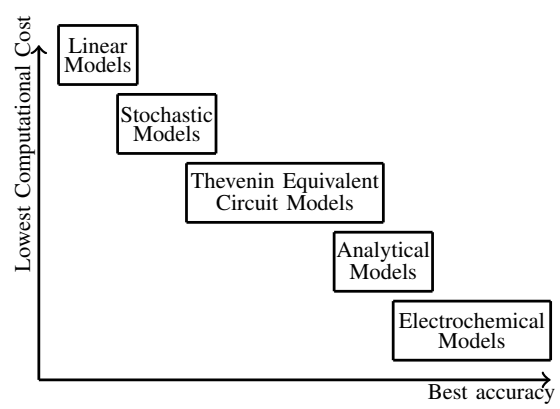

Fig. 1. Various battery model typologies are compared between model accuracy and computational cost.

simulate internally the charge and discharge processes using a battery physical model. There are a large number of models in literature, as shown in Fig. 1. Thevenin equivalent circuit model of cell should solve the compromise between computational cost and accuracy to simulate discharging process and its electrical properties. This model is described through a system of differential equations, thus a dynamic systems, where internal state evolution (SOC is a state component) of system should be monitored. Kalman Filter is the modelbased observer which estimates state of system minimizing the state mean square error. However parameters of electric battery model are not stationary, but their values depend on actual battery operative conditions. Therefore a second filter which identifies the online parameters of the battery model is used. Finally, the model adaptive Unscented Kalman Filter (UKF) [1]) method is shown in this work for online SOC estimation and model's parameters identification. Main filter's task consists of three basic steps:

- Acquisition of current and voltage data delivered by the battery.

- Upload adaptive model using Recursive Least Squares parameter identification method.

- Estimation SOC of battery model using UKF.

This work is part of the OBELICS project which has received funding from the European Unions Horizon 2020 research and innovation programme under grant agreement No. 769506. 
Every step will be described in the next sections.

\section{BATTERY MODEL}

In this work, battery model is represented by an electrical equivalent circuit of $1^{\text {st }}$ order (see Fig. 2), which is composed by the following components in series:

- Thevenin's Open Circuit Voltage (OCV): it's represented by an ideal voltage source.

- Internal resistance $\left(R_{\text {int }}\right)$ : it represents the voltage drop of the battery when it delivers/absorbs current at the load.

- One RC group $\left(R_{1}, C_{1}\right)$ : it represents the electrical dynamic behaviors of the battery, through a $1^{\text {st }}$ order's exponential transient.

The OCV value depends on the SOC of the cell, and the analytic function that relates OCV and SOC can be obtained in order that it approximates the cell discharge curve in analysis. Cell discharge curve is usually provided by the manufacturer. Accurate calibration of the OCV-SOC curve is more important for the SOC estimation using the Kalman filter [3]. Internal resistance $R_{\text {int }}$ value represents the voltage drop of the cell when it supplies/absorbs current at the load. This parameter defines battery performance and its state of health $(\mathrm{SOH})$ : maximum available charge/discharge currents and power, battery efficiency are predicted on the basis of $R_{\text {int }}$ estimation [2]. One RC group is used to represent the longterm voltage relaxation dynamics [3]. In particular, RC group describes the ion diffusion dynamics in the cell's electrolyte; further second RC group would describe the electron diffusion dynamics (faster) on the cell's electrodes, but in this work only one RC group is used. The time-continuous cell model in the state space is described by (1):

$$
\left\{\begin{aligned}
S \dot{O} C(t) & =-\frac{i(t)}{36 C_{n o m}} \\
\dot{v}_{1}(t) & =\frac{i(t)}{C_{1}}-\frac{v_{1}(t)}{R_{1} C_{1}} \\
V(t) & =f(S O C(t))-v_{1}(t)-R_{\text {int }} i(t)
\end{aligned}\right.
$$

where $O C V(t)=f(S O C(t))$ and SOC evolution is simulated by Ampere-Counting-Method [4]. Then, these equations are discretized by Eulero's approximation: let $x(t)$ a continuous function in time range $\left[t_{k}, t_{k+1}\right]$, where $k=0,1, \ldots, N$;
Eulero's approximation considers the following representation of temporal derivative of $x$ :

$$
\dot{x} \cong \frac{x\left(t_{k+1}\right)-x\left(t_{k}\right)}{t_{k+1}-t_{k}}=\frac{x\left(t_{k+1}\right)-x\left(t_{k}\right)}{T},
$$

where $T$ indicates the sample time. Combining Eq. (1), (2), cell electrical model should be described as following timediscrete dynamic system in the state space $\mathbf{x}_{k}$ :

$$
\left\{\begin{array}{c}
\mathbf{x}_{k+1}=\mathbf{A}_{k} \mathbf{x}_{k}+\mathbf{B}_{k} u_{k} \\
y_{k}=\mathbf{C}_{k} \mathbf{x}_{k}+\mathbf{D}_{k} u_{k}
\end{array}\right.
$$

where input and output $u_{k}, y_{k}$ of system (3) are respectively current and voltage delivered by cell; space's vector $\mathbf{x}_{k}=$ $\left[S O C_{k}, v_{1, k}\right]^{T}$ includes State Of Charge and voltage drop of RC group; state transition matrix $\mathbf{A}_{k}$ and input-state matrix $\mathbf{B}_{k}$ are respectively:

$$
\begin{gathered}
\mathbf{A}_{k}=\operatorname{diag}\left(\left[1, e^{-T / \tau_{1}}\right]\right), \\
\mathbf{B}_{k}=\left[-T /\left(36 C_{n o m}\right), R_{1}\left(1-e^{-T / \tau_{1}}\right)\right]
\end{gathered}
$$

where $\tau_{1}$ is the time constant of RC groups; state-output matrix $\mathbf{C}_{k}$ and input-output matrix $\mathbf{D}_{k}$ are respectively:

$$
\mathbf{C}_{k}=[f(\cdot),-1], \quad \mathbf{D}_{k}=-R_{\text {int }}
$$

The cell model represented by dynamic system (3) should be classified as follows:

- Time-Varying (TV): in [4] electrical parameters of model as $R_{\text {int }}, R_{1}, C_{1}$ depend on operating conditions of the cell and the surrounding environment conditions.

- Non-Linear (NL): analytic OCV-SOC relationship is non-linear.

\section{TEST DATA}

Five battery packs LiFePO4 with BMS installed on EV (Isuzu L35) are monitored. Nominal voltage and nominal capacity of battery pack is respectively $96 V_{d c}$ and 72 Ah: it is composed by 30 China Aviation Lithium Battery (CALB) cells of $3.2 V_{d c}$ and $72 \mathrm{Ah}$ connected in series. BMS acquires voltage and current delivered by battery pack, internal temperature of battery pack and calculates SOC every 5 seconds $(T=5 s)$.

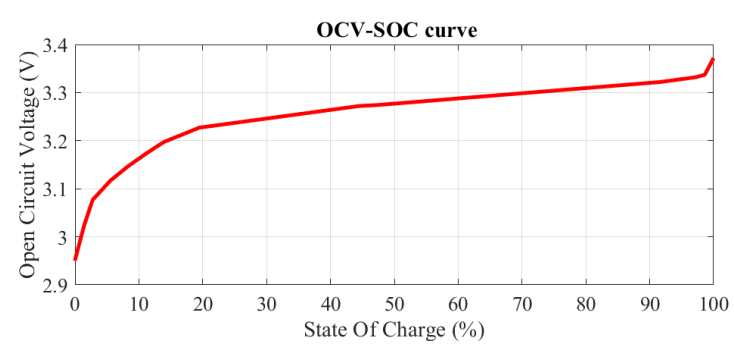

Fig. 3. Cell discharge curve of $\mathrm{LiFePo} 4$ cell (CALB).

Fig. 2. Electrical equivalent cell model. 
Battery data-set are sent on CANbus to vehicle's datalogger and memorized on micro SD (Fig. 4); further information is provided in [5]. Every day, at midnight, battery pack's data-set are uploaded to server in FTP, using 3G cellular network.

\section{ONLINE PARAMETER IDENTIFICATION}

\section{A. Arx model}

Algorithm for a polynomial and Linear In the Parameters (LIP) class of models is chosen to identify battery model's parameters. Therefore battery model is transformed in an Auto-Regressive with eXternal input (ARX) model, which is polynomial and LIP. The first step for using the ARX structure is the linearization of OCV-SOC relationship around the timevariant operating point of the cell. Since measurements are acquired every 5 seconds, the operating point should not change and SOC value remains constant during identification. So the OCV-SOC non-linear function is approximated by linear curve:

$$
O C V=\alpha_{0}+\alpha_{1} S O C
$$

where $\alpha_{0}$ and $\alpha_{1}$ change with operating point. Battery model (3) should be defined by the transfer function $G(z)$ using ZetaTransform, where current input $U(z)$ and voltage output $Y(z)$ in the frequency's domain are represented by complex variable $z$ :

$$
G(z)=\frac{Y(z)-\alpha_{0}}{U(z)}=\frac{B_{0}+B_{1} z^{-1}+B_{2} z^{-2}}{1+A_{1} z^{-1}+A_{2} z^{-2}},
$$

Transfer function's parameters are the following:

$$
\left\{\begin{array}{l}
B_{0}=R_{\text {int }} \\
B_{1}=\frac{\alpha_{1} * T}{36 C_{n o m}}+R_{1}\left(1-e^{-\frac{T}{\tau}}\right)-R_{\text {int }}\left(1+e^{-\frac{T}{\tau}}\right) \\
B_{2}=\frac{-e^{-\frac{T}{\tau}} \alpha_{1} * T}{36 C_{n o m}}-R_{1}\left(1-e^{-\frac{T}{\tau}}\right)+R_{i n t} e^{-\frac{T}{\tau}} \\
A_{1}=-1-e^{-\frac{T}{\tau}} \\
A_{2}=e^{-\frac{T}{\tau}}
\end{array}\right.
$$

Now, transfer function $G(z)$ is transformed in the $2^{\text {nd }}$ order ARX model at the step $k$ :

$$
\begin{aligned}
V_{k}= & -A_{1} V_{k-1}-A_{2} V_{k-2}+\alpha_{0}\left(1+A_{1}+A_{2}\right) \\
& -B_{0} i_{k}-B_{1} i_{k-1}-B_{2} i_{k-2}+\xi_{k}
\end{aligned}
$$

where $\xi_{k}$ indicates ARX process disturb, which is modeled as a white noise with zero mean and finite variance. From equations in (8), it's noticeable that $1+A_{1}+A_{2}=0$. This means that $\alpha_{0}$ value has not effect on voltage $V_{k}$ estimation (9), so on parameter's identification. In other terms, $\alpha_{0}$ is simply an output voltage offset which doesn't influence dynamics of battery system.

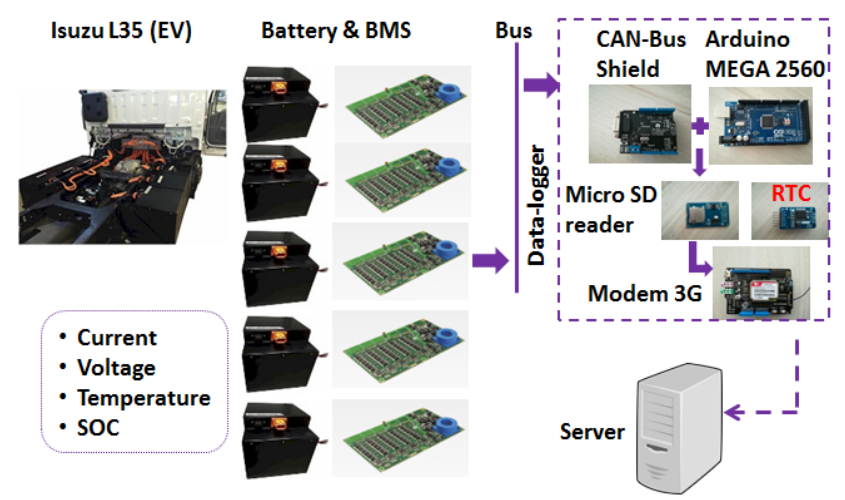

Fig. 4. Logging batteries data.

\section{B. Recursive Least Square with Forgetting Factor (RLSFF)}

Battery model (3) has been transformed in ARX model (9) which is polynomial and LIP. The parametric identification criterion with Minimum Error Prediction (PEM) is one of the best methods for online estimation of parameters of the ARX model. The prediction error of the voltage measurement $y_{k}=$ $V_{k}$ at step $k$ is defined by the following equation:

$$
\xi_{k}(\theta)=y_{k}-\varphi_{k}^{T} \theta
$$

$\varphi_{k}$ is linear regression vector at step $k$ and $\theta$ is parameter vector. ARX battery model (9) has been transformed in equation form (10). Parametric identification algorithm PEM recursively estimates the parameters of vector $\theta^{*}$ to minimize the following performance index [6]:

$$
J_{k}^{\lambda}(\theta)=\sum_{t=0}^{k} \lambda^{k-t}\left(y_{t}-\varphi_{t}^{T} \theta_{t}\right)^{2}
$$

The squares of prediction errors committed at step $k$ and in previous instances are weighted by a parameter $\lambda \in[0,1]$ called forgetting factor. Performance index (11) has been chosen so that the terms of cost $J_{k}^{\lambda}$ are weighted according to the distance from the current time $k T$. In this way, parametric identification algorithm is able to recursively estimate parameters effectively by varying of actual operating conditions of the battery. Algorithm considers more recent cost terms when $\lambda$ is smaller. Typical $\lambda$ values are $0.995,0.99,0.98$. Therefore, ARX model's parameter $\theta$ identification is computed at each step $k$, minimizing actual performance index (9). Given $\left[\varphi_{k}, \lambda, \theta_{k-1}^{*}, \phi_{k-1}\right]$, where estimation error of $\theta_{k-1}$ is represented by the covariance matrix $\phi_{k-1}$; acquiring voltage measurement $y_{k}$, RLSFF parameter identification algorithm consists of three simple matrix equations:

$$
\begin{gathered}
\Phi_{\lambda, k}=\frac{1}{\lambda}\left[\Phi_{\lambda, k-1}-\frac{\Phi_{\lambda, k-1} \varphi_{k} \varphi_{k}^{T} \Phi_{\lambda, k-1}}{\lambda+\varphi_{k}^{T} \Phi_{\lambda, k-1} \varphi_{k}}\right] \\
L_{\lambda, k}=\frac{\Phi_{\lambda, k-1} \varphi_{k}}{\lambda+\varphi_{k}^{T} \Phi_{\lambda, k-1} \varphi_{k}} ; \\
\theta_{k}^{*}=\theta_{k-1}^{*}+L_{\lambda, k}\left(y_{k}-\varphi_{k}^{T} \theta_{k-1}^{*}\right) ;
\end{gathered}
$$



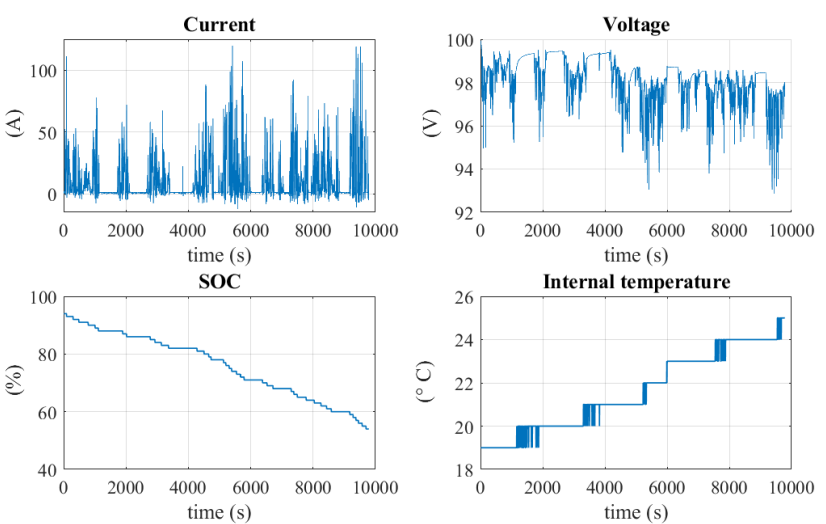

Fig. 5. Data-set of battery pack 1 during a test.

These operations follow a Bayesian estimation approach. Indeed $\theta_{k}^{*}$ identification is the result of the sum of the $\theta_{k-1}^{*}$ identification made in the previous step $k-1$ and an additional information given by the $y_{k}$ measurement. This last information is acquired at current time instant $k$ and is weighted by a gain $L_{\lambda, k}$ which depends on the covariance matrix $\Phi_{\lambda, k}$ of estimation error which is obtained at step $k$. Covariance matrix $\Phi_{\lambda, k}$ is defined in [6] as:

$$
\Phi_{\lambda, k} \doteq\left(\sum_{t=0}^{k} \lambda^{k-t} \varphi_{t} \varphi_{t}^{T}\right)^{-1}
$$

Covariance matrix returns smaller values when the matrix $\sum_{t=0}^{k} \varphi_{t} \varphi_{t}^{T}$ returns larger values, i.e. when data-set of measures $u_{[0, k]}$ and $y_{[0, k]}$ is sufficiently informative. It happens when battery process is in persistent excitement, i.e., when $\mathrm{EV}$ is moving.

\section{Results}

RLSFF method is used for online identification of Thevenin circuit model's parameters $\left(R_{\text {int }}, R_{1}, C_{1}, \alpha_{1}\right)$ shown in Fig. 2 . The effectiveness of this algorithm has been verified based on monitoring of five LiFePO4 battery packs installed on a working EV in March-April 2017 (43 tests are executed). Matlab software is used to implement and run RLSFF, based on current, voltage input data acquired from data-logger, at the sample time $T=5 \mathrm{~s}$. An example of this data-set is shown in Fig. 5, and results of online parameter identification based on this data-set is shown in Fig. 6. Despite the manufacture of five battery packs is the same, we observe that battery pack's parameters doesn't clearly evolve in time with equal values. The causes are two: BMS master manages the equalization requiring different intensity of current for each battery; moreover batteries are placed in different positions of vehicle, consequently they are exposed under different temperatures. To give a summary of estimations of circuit model parameters, mean and standard deviation (std) of $R_{\text {int }}, R_{1}, \tau_{1}$ (where $\tau$ is the time constant of RC group) are illustrated, during all monitoring tests performed in Table I.
TABLE I

SUMMARY OF ELECTRICAL EQUIVALENT BATTERY MODEL PARAMETERS

\begin{tabular}{|c|c|c|c|}
\hline & $R_{\text {int }}(m \Omega)$ & $R_{1}(m \Omega)$ & $\tau_{1}(s)$ \\
\hline Pack 1 & $38.72 \pm 4.70$ & $4.81 \pm 2.69$ & $3.44 \pm 0.58$ \\
Pack 2 & $38.76 \pm 5.07$ & $4.01 \pm 3.08$ & $3.48 \pm 0.60$ \\
Pack 3 & $37.98 \pm 4.94$ & $4.58 \pm 2.04$ & $3.53 \pm 0.59$ \\
Pack 4 & $41.21 \pm 5.23$ & $5.24 \pm 2.45$ & $3.54 \pm 0.52$ \\
Pack 5 & $39.67 \pm 5.15$ & $4.77 \pm 2.24$ & $3.44 \pm 0.62$ \\
\hline
\end{tabular}

It's noticeable the importance of RLSFF filter: battery model parameters vary depending on the environmental conditions (as temperature) and operative conditions (as SOC, aging, etc.). From this consideration, it would be required look-up table implementations which relate parameters with temperature and SOC. In particular, for internal resistance $R_{i n t}$, is realized a surface function $\Upsilon(S O C, T)$, which returns the value of the internal resistance $R_{\text {int }}$ in function of the SOC and the internal temperature $T$, shown in Fig. 7. This function is obtained using data acquired from data-logger during monitoring and the Curve Fitting Toolbox of Matlab. Results obtained indicate that $R_{\text {int }}$ value increases when temperature decreases and at low SOC values, justifying electro-chemical behavior of battery, due in particular to ionic conductivity and the number of electrical active elements in the electrolyte of each single cell.
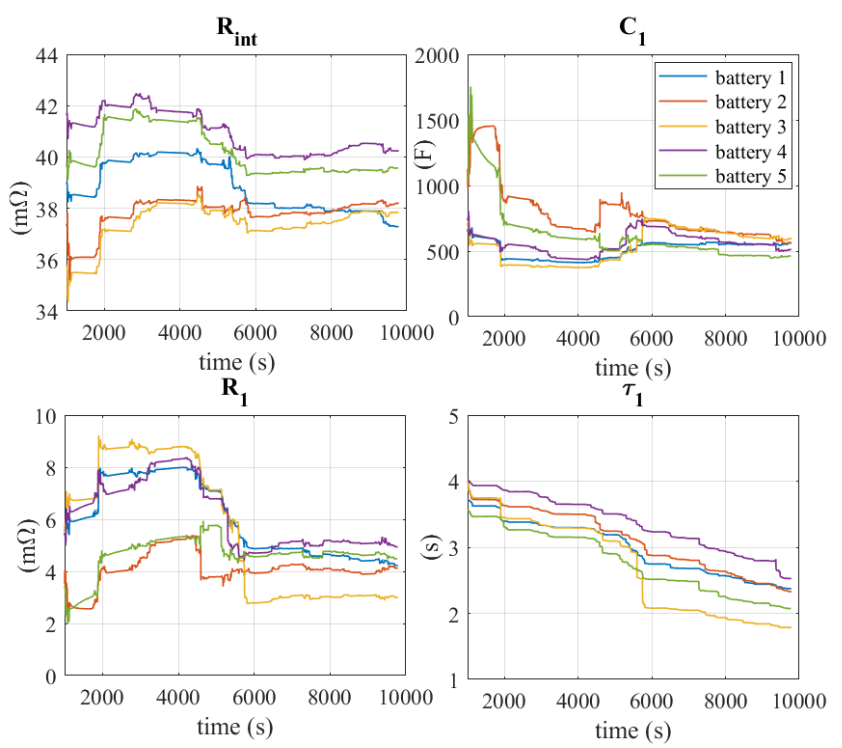

Fig. 6. Electrical equivalent battery model parameter identification.

TABLE II

RLSFF PERFORMANCE (RELATIVE TO $96 V_{\text {nom }}$ BATTERY PACKS).

\begin{tabular}{|l|l|l|}
\hline & mean RMSE (\%) & std RMSE (\%) \\
\hline Pack 1 & $0.169 \%$ & $0.058 \%$ \\
Pack 2 & $0.175 \%$ & $0.062 \%$ \\
Pack 3 & $0.167 \%$ & $0.066 \%$ \\
Pack 4 & $0.166 \%$ & $0.053 \%$ \\
Pack 5 & $0.169 \%$ & $0.058 \%$ \\
\hline
\end{tabular}




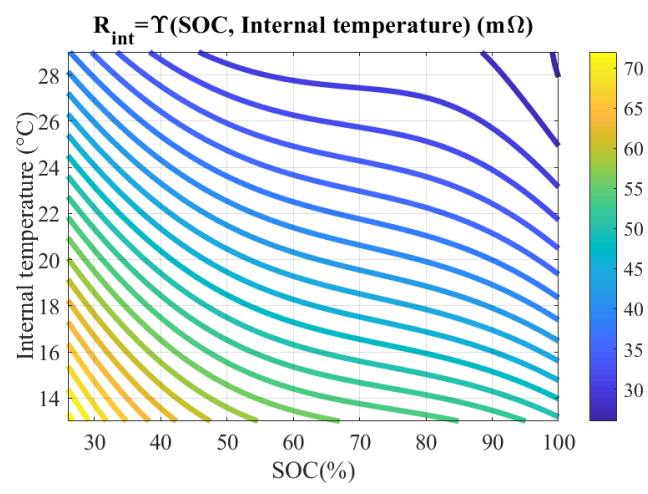

Fig. 7. Analytical function $\Upsilon$ : contour plot.

Finally, the effectiveness of RLSFF is evaluated performing 100 Montecarlo trials for each test, varying initial conditions of estimated model's parameters $\left(R_{i n t}, R_{1}, C_{1}\right)$. Root Mean Square error moments (mean and standard deviation) are computed for each pack, shown in Table II.

\section{Model Adaptive Kalman Filter}

\section{A. Presentation}

BMS needs to have accurate estimation of SOC. In battery system (3), it's represented as an element of state vector $\mathbf{x}_{k}$. Unscented Kalman Filter (UKF) is an optimum observer for non-linear systems, which follow a Bayesian estimation approach. Assuming that equations of battery model (3) are influenced by the process and measurement noise (respectively $\left.\mathbf{w}_{k}, v_{k}\right)$, and their properties are as follows:

$$
\begin{aligned}
& \left\{\begin{array}{l}
\mathbf{x}_{k+1}=\mathbf{A}_{k} \mathbf{x}_{k}+\mathbf{B}_{k} u_{k}+\mathbf{w}_{k} \\
y_{k}=\mathbf{C}_{k} \mathbf{x}_{k}+\mathbf{D}_{k} u_{k}+v_{k}
\end{array}\right. \\
& \mathbf{w}_{k} \sim N\left(0, \mathbf{Q}_{k} \succeq 0\right), v_{k} \sim N\left(0, \mathbf{R}_{k} \succeq 0\right), \\
& \mathbf{w}_{k} \perp v_{k} \perp \mathbf{x}_{0}
\end{aligned}
$$

From previous section, battery model parameters are not stationary; consequently UKF algorithm recursively computes SOC estimation after that battery adaptive model is uploaded using RLSFF filter.

\section{B. Unscented Kalman Filter (UKF)}

Unscented Kalman Filter uses the same framework of Kalman Filter for linear systems, and it replaces the tedious linearization method of non-linear function in an equilibrium point from the Extended Kalman Filter (EKF) with a deterministic sampling method of the equilibrium point distribution. Unscented Transform function (UT) is used to propagate the random variable moments of equilibrium point (mean $\hat{\mathbf{x}}$, covariance matrix $\mathbf{P}_{\mathbf{x}}$ ) through the non-linear function of battery model. Given the variable random $\mathbf{x}_{k}$, which mean and covariance matrix are known:

$$
\left\{\begin{array}{l}
\hat{\mathbf{x}}_{k}=E\left[\mathbf{x}_{k}\right]=\left[S O C_{k}, v_{1, k}\right]^{T} \\
\mathbf{P}_{\mathbf{x}, k}=E\left[\left(\mathbf{x}_{k}-\hat{\mathbf{x}}_{k}\right)\left(\mathbf{x}_{k}-\hat{\mathbf{x}}_{k}\right)^{T}\right]
\end{array}\right.
$$

UT approximates the variable random distribution with a finite number of samples, called $\sigma-$ point. In [1] it is shown that $n+1$ $\sigma-$ points are sufficient, even if, for symmetry reasons, usually $2 n+1$ are used, indicated with $\mathbf{x}_{i}$ for $i=-n, \ldots, 0, \ldots, n$, with relative weights $w_{i}$. The equality of mean and covariance of distribution sampled by the $\sigma$-point with mean and covariance of $\mathbf{x}_{k}$ imposes the following equations:

$$
\left\{\begin{array}{l}
\hat{\mathbf{x}}_{k}=\sum_{i=-n}^{n} w_{i} \mathbf{x}_{i} \\
\mathbf{P}_{\mathbf{x}, k}=\sum_{i=-n}^{n} w_{i}\left(\mathbf{x}_{i}-\hat{\mathbf{x}}_{k}\right)\left(\mathbf{x}_{i}-\hat{\mathbf{x}}_{k}\right)^{T}
\end{array}\right.
$$

Therefore, $\sigma$-point which sample distribution of battery model state $\mathbf{x}_{k}$ are generated by equations in (18). In literature there are many approach for $\sigma$-point generation [1]. Given non-linear transformation $\mathbf{y}_{k}=\mathbf{g}\left(\mathbf{x}_{k}\right)$, i.e.:

$$
\mathbf{x}_{k} \sim\left(\hat{\mathbf{x}}_{k}, \mathbf{P}_{\mathbf{x}, k}\right) \stackrel{\mathbf{g}(\cdot)}{\longrightarrow} \mathbf{y}_{k} \sim\left(\hat{\mathbf{y}}_{k}, \mathbf{P}_{\mathbf{y}, k}\right)
$$

UT function propagates $\sigma$-point through non-linear function:

$$
\mathbf{y}_{i}=\mathbf{g}\left(\mathbf{x}_{i}\right), \quad i=-n, \ldots, 0, \ldots, n
$$

From last equation, mean, covariance and cross-covariance matrix moments of output $\mathbf{y}_{k}$ distribution are approximately computed by following equations:

$$
\left\{\begin{array}{l}
\hat{\mathbf{y}}_{k}=\sum_{i=-n}^{n} w_{i} \mathbf{y}_{i} \\
\mathbf{P}_{\mathbf{y}, k}=\sum_{i=-n}^{n} w_{i}\left(\mathbf{y}_{i}-\hat{\mathbf{y}}_{k}\right)\left(\mathbf{y}_{i}-\hat{\mathbf{y}}_{k}\right)^{T} \\
\mathbf{P}_{\mathbf{x y}, k}=\sum_{i=-n}^{n} w_{i}\left(\mathbf{x}_{i}-\hat{\mathbf{x}}_{k}\right)\left(\mathbf{y}_{i}-\hat{\mathbf{y}}_{k}\right)^{T}
\end{array}\right.
$$

In this work, battery model (16) presents non-linear function in the output equation, exactly the OCV-SOC curve shown in Fig. 3. As already mentioned, Unscented Kalman Filter uses the same framework of Kalman Filter for linear systems; consequently the UT function is used in the classical Kalman correction phase:

$$
\left\{\begin{array}{l}
\hat{\mathbf{x}}_{k \mid k}=\hat{\mathbf{x}}_{k \mid k-1}+\mathbf{L}_{k}\left(\mathbf{y}_{k}-\hat{\mathbf{y}}_{k \mid k-1}\right) \\
\mathbf{P}_{\mathbf{x}, k \mid k}=\mathbf{P}_{\mathbf{y}, k \mid k-1}-\mathbf{L}_{k} \mathbf{P}_{\mathbf{x y}, k \mid k-1}
\end{array}\right.
$$

where the moments of battery model voltage $\left[\hat{\mathbf{y}}_{k \mid k-1}, \mathbf{P}_{\mathbf{y}, k \mid k-1}, \mathbf{P}_{\mathbf{x y}, k \mid k-1}\right]$ and Kalman gain matrix $\mathbf{L}_{k}$ are computed using UT function and the OCV-SOC non-linear curve, processing the previous state prediction $\left[\hat{\mathbf{x}}_{k \mid k-1}, \mathbf{P}_{\mathbf{x}, k \mid k-1}\right]$.

\section{Results}

Model adaptive Unscented Kalman filter (defined RLSFFUKF) is composed by the RLSFF filter which, acquiring each sample time $k T$ current $\left(i_{k}\right)$ and voltage $\left(v_{k}\right)$ data delivered by the battery, identifies and uploads battery model (16) to UKF, which computes online SOC estimation. 


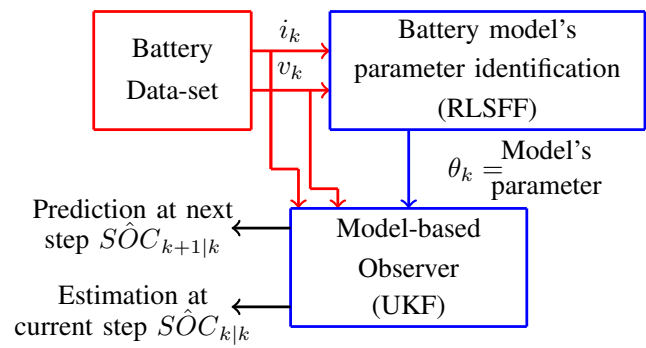

Fig. 8. RLSFF-UKF block system: red stays for acquired measurement and blue stays for computed data-set.

TABLE III

SUMMARY OF SOC ESTIMATION RESULTS COMPUTED BY RLSFF-UKF.

\begin{tabular}{|c|c|c|c|}
\hline \multicolumn{2}{|c|}{ max. SOC error } & \multicolumn{2}{c|}{ mean SOC error } \\
\hline$($ mean \%) & $($ std \%) & $($ mean \%) & (std \%) \\
\hline $1.23 \%$ & $0.53 \%$ & $0.52 \%$ & $0.33 \%$ \\
\hline
\end{tabular}

Matlab software is used to implement the filter and estimation of the state evolution is elaborated based on current, voltage acquired from data-logger, at the sample time $T=5 \mathrm{~s}$. Performances of model adaptive UKF are evaluated computing maximum and mean absolute of SOC error, i.e. the difference between SOC estimated by RLSFF-UKF and SOC calculated by vehicle's BMS. These results in summary form (mean, std) are shown in Table III, for battery pack 1, during March-April 2017 (43 tests are executed).

\section{CONCLUSIONS}

The model adaptive Unscented Kalman filter has been applied to the identification of the battery parameters and SOC estimation. The battery has been modeled by a Thevenin equivalent electrical model, which should solve trade-off between accuracy and computational complexity in simulating electrical battery behaviors. Then, a model-based observer has been synthesized and implemented on Matlab. This filter is composed by two filters in series: a recursive least squares with forgetting factor (RLSFF) filter which estimates online parameters and uploads during each sample time electrical battery circuit, in order that Kalman Filter (exactly UKF) recursively estimates model states, as SOC. Performances of the proposed filter have been verified based on monitoring five $\mathrm{LiFePO} 4$ battery packs installed on working $\mathrm{EV}$ in March, April 2017. Results shown in Table II and III proof optimal performances of the filter. However UKF elaborates an accurate SOC estimation if OCV-SOC curve provided by manufacturer is accurate and initial SOC estimation is close to its exact value. Moreover it's important to specify that SOC accuracy of BMS is $5 \%$ due to the low accuracy of current sensors; so it would be advisable to calibrate the algorithm on tests carried out in laboratory with ad hoc current sensors. An example of a similar work is shown in [3]. Despite the manufacture of five battery packs is the same, in Table I is noticeable that parameters aren't exactly equal between each
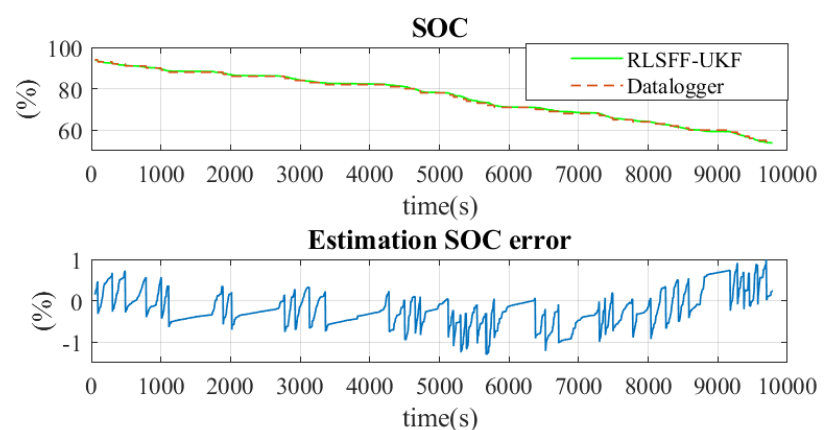

Estimation output voltage error

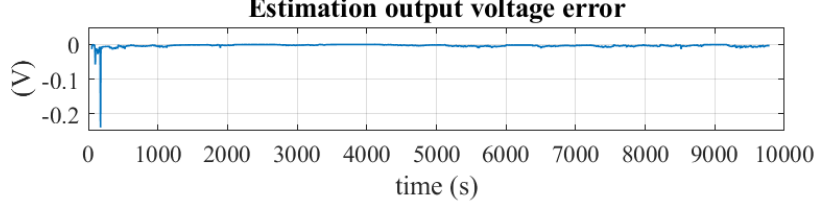

Fig. 9. Results of model adaptative UKF based on test shown in Fig. 5 (mean estimation output voltage error $2.9 \mathrm{mV}$ ).

battery pack. Indeed BMS master manages the equalization requiring different intensity of current for each battery; moreover, batteries are placed in different positions of vehicle, consequently they are exposed under different temperatures. In addition, it's possible that the cells in each battery pack are slightly different due to manufacturing tolerances. Finally, it's noticeable that in Table I parameters are not stationary but instantly depend by operating and weather conditions of battery. For example, battery internal resistance increases at low SOC and when surrounding temperature decreases, as justified by electro-chemical behavior of it. Possible future developments are implementation of this algorithm in real industrial BMS for Lithium-Ion batteries.

\section{ACKNOWLEDGMENT}

The present work is using data coming from electrical vehicle belonging to Gruppo Pretto company (Ponsacco, Italy); our special thanks to the leader of this company Alessandro Pretto for his support and collaboration.

\section{REFERENCES}

[1] S. Julier and J. Uhlmann, Unscented filtering and nonlinear estimation Proceedings of the IEEE, vol. 92, no. 3, pp. 401-422, 2004.

[2] P. Shen, M. Ouyang, L. Lu, J. Li, The Co-estimation of State of Charge, State of Health, and State of Function for Lithium-Ion Batteries in Electric Vehicles, IEEE Transactions on vehicular technology, Vol. 67, No. 1, January 2018.

[3] T. Huria, G. Ludovici, G. Lutzemberger, "State of charge estimation of high power lithium iron phosphate cells", Journal of Power Sources, Volume 249, Pages 92-102, 1 March 2014.

[4] F. Baronti, W. Zamboni, N. Femia, H. Rahimi-Eichi , R. Roncella, S Rosi, R. Saletti, and M.-Y. Chow, "Parameter identification of Li-Po batteries in electric vehicles: A comparative study", 2013 IEEE International Symposium on Industrial Electronics, Taipei, Taiwan, 2013, pp. 1-7.

[5] L. Pugi, M. Pagliai, A. Nocentini, G. Lutzemberger, A. Pretto, ’Design of a hydraulic servo-actuation fed by a regenerative braking system", Applied Energy, Volume 187, Pages 96-115, 1 February 2017.

[6] L. Ljung, "System Identification - Theory for the User", Prentice Hall, 1999. 